\title{
Resolving the vacuum fluctuations of an optomechanical system using an artificial atom
}

\author{
F. Lecocq ${ }^{\star}$, J. D. Teufel, J. Aumentado and R. W. Simmonds
}

\begin{abstract}
Heisenberg's uncertainty principle results in one of the strangest quantum behaviours: a mechanical oscillator can never truly be at rest. Even at a temperature of absolute zero, its position and momentum are still subject to quantum fluctuations ${ }^{1,2}$. However, direct energy detection of the oscillator in its ground state makes it seem motionless ${ }^{1,3}$, and in linear position measurements detector noise can masquerade as mechanical fluctuations ${ }^{4-7}$. Thus, how can we resolve quantum fluctuations? Here, we parametrically couple a micromechanical oscillator to a microwave cavity to prepare the system in its quantum ground state $e^{8,9}$ and then amplify the remaining vacuum fluctuations into real energy quanta ${ }^{10}$. We monitor the photon/phonon-number distributions using a superconducting qubit ${ }^{11-13}$, allowing us to resolve the quantum vacuum fluctuations of the macroscopic oscillator's motion. Our results further demonstrate the ability to control a long-lived mechanical oscillator using a non-Gaussian resource, directly enabling applications in quantum information processing and enhanced detection of displacement and forces.
\end{abstract}

Cavity optomechanical systems have emerged as an ideal testbed for exploring the quantum limits of linear measurement of macroscopic motion ${ }^{2}$, as well as a promising new architecture for performing quantum computations. In such systems, a light field reflecting off a mechanical oscillator acquires a position-dependent phase shift and reciprocally, it applies a force onto the mechanical oscillator. This effect is enhanced by embedding the oscillator inside a high-quality-factor electromagnetic cavity. Numerous physical implementations exist, both in the microwave and optical domains, and have been used to push the manipulation of macroscopic oscillators into the quantum regime, demonstrating laser cooling to the ground state of motion ${ }^{8,14}$, coherent transfer of itinerant light fields into mechanical motion ${ }^{9,15}$, or their entanglement ${ }^{10}$. Thus far, linear position measurements have provided evidence for the quantization of light fields through radiation pressure shot noise $\mathrm{e}^{16,17}$ and mechanical vacuum fluctuations through motional sideband asymmetries ${ }^{4-7}$. However, the use of only classical and linear tools has restricted most optomechanical experiments to the manipulation of Gaussian states.

The addition of a strong nonlinearity, such as an atom, has fostered tremendous progress towards exquisite control over nonGaussian quantum states of light fields and atomic motion ${ }^{11,12}$. First developed in the context of cavity quantum electrodynamics (cQED), these techniques are now widely applied to engineered systems, such as superconducting quantum bits (qubits) and microwave resonant circuits $^{13,18-21}$. In a pioneering cQED-type experiment, a qubit resonantly coupled to a high-frequency mechanical oscillator ${ }^{3}$ allowed for the control of a single-phonon Fock state. However, short energy lifetimes of the mechanical oscillator and the qubit have slowed any further progress.
Here, we go beyond just cQED by introducing cavity optomechanical interactions. Our unique architecture incorporates an artificial atom-a superconducting qubit ${ }^{22}$-into a circuit cavity electromechanical system ${ }^{23}$, on a single chip. Here, a low-frequency, high-quality-factor mechanical oscillator strongly interacts with the microwave cavity photons. The qubit-cavity interaction realizes a non-classical emitter and detector of photons, thus providing an essential nonlinear resource for the deterministic control of long-lived mechanical quantum states. We demonstrate the potential of such an architecture by measuring the quantum vacuum fluctuations inherently present in the motion of a macroscopic oscillator.

A microwave cavity is the central element of this architecture (in blue in Fig. 1a). It is a linear inductor-capacitor (LC) resonator formed by a coil inductor and a mechanically compliant vacuum-gap capacitor ${ }^{23,24}$. First, the intra-cavity electromagnetic field is coupled by means of radiation pressure to the vibrational mode of the compliant capacitor (in red in Fig. 1). Second, the microwave cavity is capacitively coupled to a phase qubit (in green in Fig. 1). A phase qubit is formed from a Josephson junction in parallel with an LC oscillator, and it behaves like a nonlinear resonator at the single-quantum level, that is, an artificial atom $^{22}$. To a good approximation the phase qubit can be operated as a two-level system whose transition frequency $\omega_{\mathrm{qb}}$ can be widely tuned in situ by applying an external flux, such that $9 \mathrm{GHz} \leq \omega_{\mathrm{qb}} / 2 \pi \leq 13.5 \mathrm{GHz}$. The microwave cavity and the fundamental flexural mode of the capacitor are two harmonic oscillators with resonance frequencies of respectively $\omega_{c} / 2 \pi=10.188 \mathrm{GHz}$ and $\Omega_{\mathrm{m}} / 2 \pi=15.9 \mathrm{MHz}$.

The qubit and the cavity are both electrical circuits with quantized energy levels, sharing a voltage through the coupling capacitor. On resonance, $\Delta_{\mathrm{qb}}=\omega_{\mathrm{qb}}-\omega_{\mathrm{c}}=0$, the interaction between the qubit and the cavity is well described by the Jaynes-Cummings Hamiltonian $\mathcal{H}_{\mathrm{jc}}=\hbar J\left(\hat{a} \hat{\sigma}_{+}+\hat{a}^{\dagger} \hat{\sigma}_{-}\right)$. Here, $\hat{\sigma}_{+}\left(\hat{\sigma}_{-}\right)$is the raising (lowering) operator for the qubit, $\hat{a}^{\dagger}(\hat{a})$ is the creation (annihilation) operator for cavity photons and $J$ is the capacitive coupling strength. $\mathcal{H}_{\mathrm{jc}}$ describes the exchange of a single quantum between the qubit and the cavity at a rate $2 J$. In the strong-coupling regime, when the coupling strength $J$ overcomes the decoherence rates of the qubit $\gamma_{\mathrm{qb}}$ and the cavity $\kappa$, that is, $J>\left(\gamma_{\mathrm{qb}}, \kappa\right)$, the system hybridizes, leading to the well-known vacuum Rabi splitting, measured spectroscopically in Fig. 1e. The qubit-cavity interaction can be effectively turned off by detuning the qubit.

The position of the mechanical oscillator modulates the cavity resonance frequency and thus, the energy stored in the cavity. As a result, the microwave photons apply a force on the mechanical oscillator. This interaction is described by the radiation pressure Hamiltonian $^{2} \mathcal{H}_{\mathrm{rp}}=\hbar G \hat{n}_{\mathrm{c}} \hat{x}$, where $G=\mathrm{d} \omega_{\mathrm{c}} / \mathrm{d} x, \hat{n}_{\mathrm{c}}=\hat{a}^{\dagger} \hat{a}$ is the cavity photon number and $\hat{x}=x_{\mathrm{zpf}}\left(\hat{b}^{\dagger}+\hat{b}\right)$ is the oscillator's position. Here, $x_{\text {zpf }}$ is the oscillator's zero-point fluctuation and $\hat{b}^{\dagger}(\hat{b})$ is the creation 

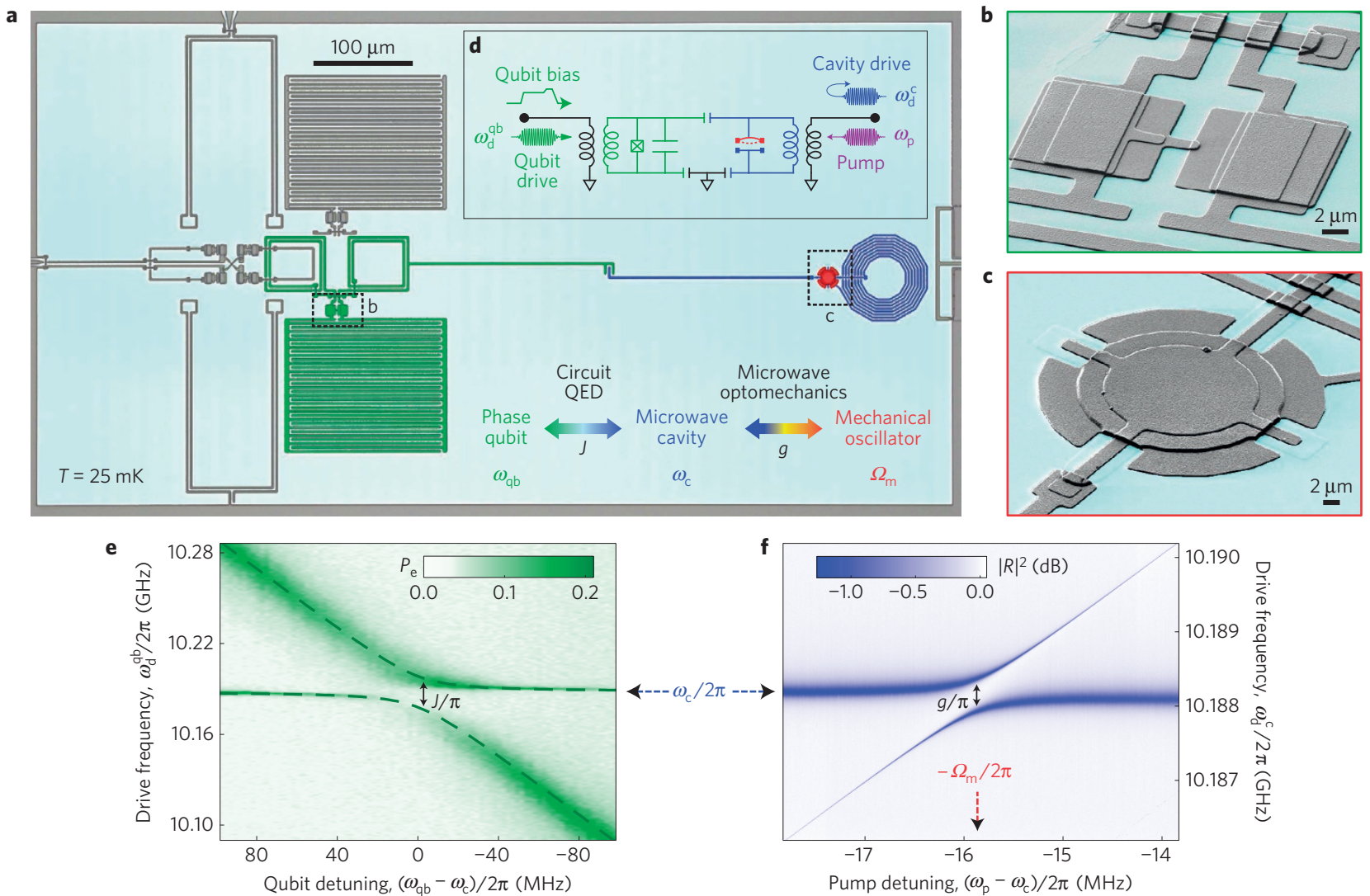

Figure 1 | Device description and strong-coupling regime. a, False-colour optical micrograph of the device. Aluminium is in grey and sapphire in light blue. The phase qubit is in green; the microwave cavity in blue; and the mechanically compliant capacitor in red. b,c, False-colour scanning electron micrograph of the qubit's Josephson junction and of the mechanical oscillator, respectively. d, Equivalent circuit diagram. e, Qubit-cavity vacuum Rabi splitting. Population of the qubit's excited state $P_{\mathrm{e}}$, in green, as a function of the drive frequency and of the qubit detuning with respect to the microwave cavity. The avoided crossing is a clear signature of the single-photon strong-coupling regime. From a fit with theory we extract the qubit/cavity coupling rate $J / 2 \pi=12.5 \mathrm{MHz}$. f, Cavity-oscillator normal-mode splitting. Reflected power in blue as a function of the drive and pump frequencies. The drive is applied near the cavity resonance and the pump is applied near $\Delta_{\mathrm{p}}=-\Omega_{\mathrm{m}}$. Here, the pump strength is set to $n_{\mathrm{p}} \approx 5 \times 10^{5}$. The normal-mode splitting observed in the cavity-driven response corresponds to the hybridization of the cavity and mechanical modes in the driven strong-coupling regime. From a fit with theory we extract $\kappa / 2 \pi=163 \mathrm{kHz}, \Gamma_{\mathrm{m}} / 2 \pi=150 \mathrm{~Hz}$ and $g=2 \pi \times 242 \mathrm{kHz}>\left(\bar{n}_{\mathrm{m}} \Gamma_{\mathrm{m}}, \kappa\right)$, where $\bar{n}_{\mathrm{m}} \approx 32$ at $T \approx 25 \mathrm{mK}$.

(annihilation) operator for mechanical phonons. The force applied by a single photon onto the mechanical oscillator is typically weak, with $g_{0}=G x_{\mathrm{zpf}} \ll\left(\bar{n}_{\mathrm{m}} \Gamma_{\mathrm{m}}, \kappa\right)$, where $\bar{n}_{\mathrm{m}}$ is the equilibrium thermal occupancy of the oscillator and $\Gamma_{\mathrm{m}}$ is its intrinsic relaxation rate. However, the total force increases significantly with the intensity of the intra-cavity field. In the presence of a strong coherent microwave pump of frequency $\omega_{\mathrm{p}}$, the optomechanical interaction is linearized and takes two different forms depending on the pump-cavity detuning $\Delta_{\mathrm{p}}=\omega_{\mathrm{p}}-\omega_{\mathrm{c}}$ (Supplementary Information). When $\Delta_{\mathrm{p}}=-\Omega_{\mathrm{m}}$, the annihilation of a mechanical phonon can up-convert a pump photon into a cavity photon, mediating a 'beamsplitter' interaction, $\mathcal{H}_{-}=\hbar g\left(\hat{a} \hat{b}^{\dagger}+\hat{b} \hat{a}^{\dagger}\right)$. This results in the coherent exchange of the cavity and mechanical states at a rate $2 g$, where $g=g_{0} \sqrt{n_{\mathrm{p}}}$ is the enhanced optomechanical coupling and $n_{\mathrm{p}}$ is the pump strength expressed in terms of the average number of intra-cavity photons. When $\Delta_{\mathrm{p}}=+\Omega_{\mathrm{m}}$, pump photons are downconverted into correlated photon-phonon pairs, mediating a 'twomode squeezer' interaction, $\mathcal{H}_{+}=\hbar g\left(\hat{a}^{\dagger} \hat{b}^{\dagger}+\hat{b} \hat{a}\right)$. This results in the amplification and entanglement of the cavity field and the mechanical motion ${ }^{10}$, at a rate $2 g$. The hallmark for entering the strong-coupling regime in our device, $g>\left(\Gamma_{\mathrm{m}}, \kappa\right)$, is the hybridization and normal-mode splitting induced by a strong beamsplitter interaction ${ }^{23}$, as measured through the cavity-driven response in Fig. 1f. Finally, with a lifetime of the mechanical oscillator's ground state, $1 / \bar{n}_{\mathrm{m}} \Gamma_{\mathrm{m}} \approx 33 \mu \mathrm{s}$, much longer than the cavity lifetime, $1 / \kappa \approx 1 \mu \mathrm{s}$, this device is in the quantumcoherent regime ${ }^{15}$.

Measurements in the frequency domain provide an extensive characterization of the device's parameters; however, they probe only the steady state of the system, in equilibrium with the thermal environment. In the next two paragraphs, we will show that time-domain protocols enable: the preparation of non-classical cavity states and the measurement of the intra-cavity photonnumber distribution ${ }^{11-13}$; and coherent state transfer by frequency conversion and entanglement by parametric amplification between the microwave cavity and the mechanical oscillator ${ }^{25,26}$.

The out-of-equilibrium dynamics between the phase qubit and the cavity are shown in Fig. 2. First, in Fig. 2a,b we perform the first basic block of the Law-Eberly protocol ${ }^{27}$. We initialize the qubit in the excited state using a resonant microwave pulse, then tune the qubit into resonance with the cavity for an interaction time $\tau$ and measure the qubit population $P_{\mathrm{e}}$ (using a destructive single-shot readout). The coupled system undergoes vacuum Rabi oscillations at a single frequency $J / \pi$ and after half a cycle the cavity is prepared in a single-photon Fock state. Next, in Fig. $2 c-f$, we exploit the well-known scaling of the Rabi frequency with the cavity Fock state number to measure the intra-cavity photon-number distribution ${ }^{13}$. We initialize the cavity in either a coherent state or a thermal state, parametrized by the average photon occupancy $\left\langle\hat{a}^{\dagger} \hat{a}\right\rangle$. When the qubit is tuned into resonance, each initial distribution 
a

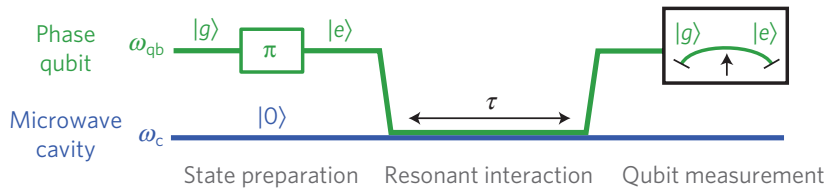

b

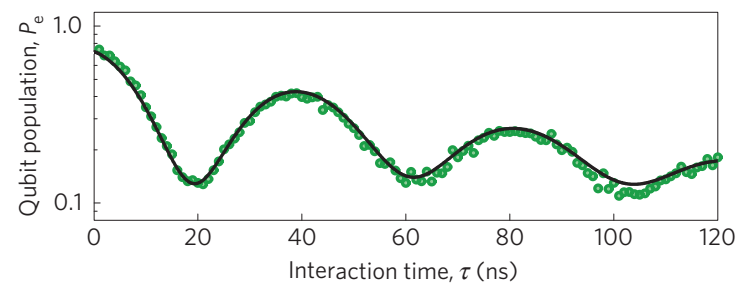

C

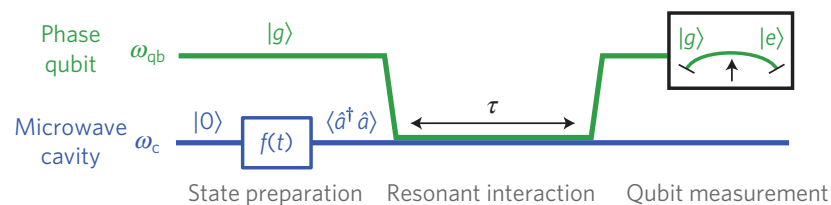

d

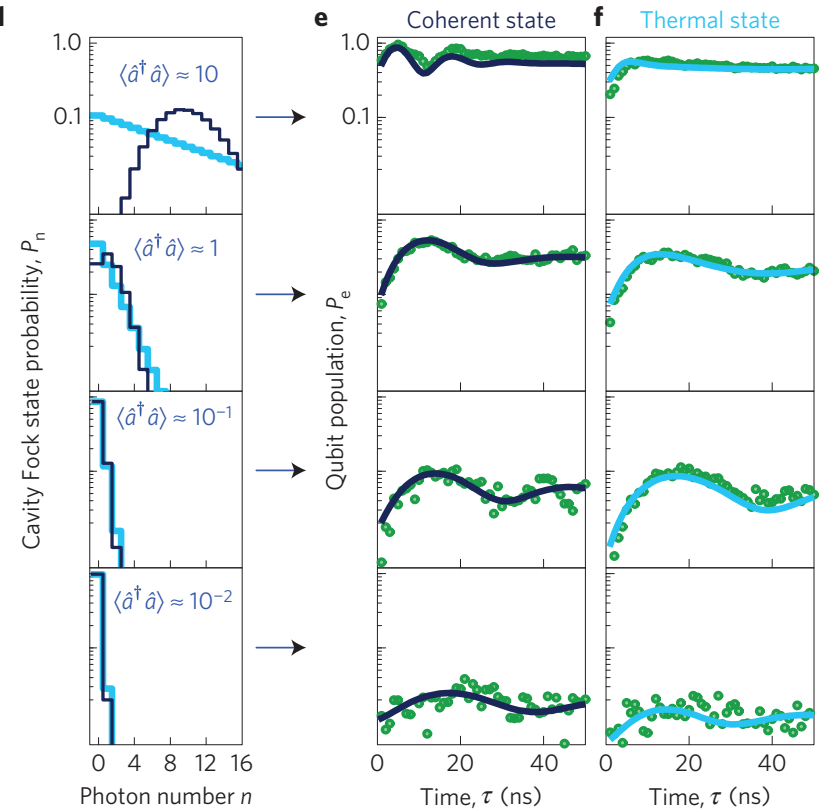

Figure 2 | Cavity state preparation and readout. a, Sequence diagram for the preparation of a single-photon Fock state in the cavity. The qubit is prepared in the first excited state $|e\rangle$, with a $75 \%$ efficiency, and then interacts resonantly with the cavity for a time $\tau$ before the qubit state is measured. $\mathbf{b}$, The population of the qubit $P_{\mathrm{e}}$ plotted as a function of the interaction time $\tau$. The black line is a fit to a master equation prediction (Supplementary Information). c, Sequence diagram for the readout of the cavity state. The cavity is prepared in a coherent (or thermal) state by driving it with a coherent tone (or with white noise). d, The corresponding intra-cavity photon distributions for four drive amplitudes, parametrized by the average cavity occupancy $\bar{n}_{\mathrm{c}}$. The distributions for thermal and coherent states are respectively in bright and dark blue. e,f, For each drive amplitude, the evolution of the qubit population $P_{\mathrm{e}}$ is plotted in green for the coherent states (e), and for the thermal states $(\mathbf{f})$. The solid lines are a fit to a master equation prediction.

(Fig. 2d) produces a distinct time evolution of $P_{\mathrm{e}}(\tau)$ (Fig. 2e,f), in good agreement with simulations that include all sources of decoherence and where the average photon number $\bar{n}_{\mathrm{c}}$ is the only free parameter (Supplementary Information). We resolve the cavity occupancy down to $\left\langle\hat{a}^{\dagger} \hat{a}\right\rangle \approx 0.02$ and have the ability to distinguish

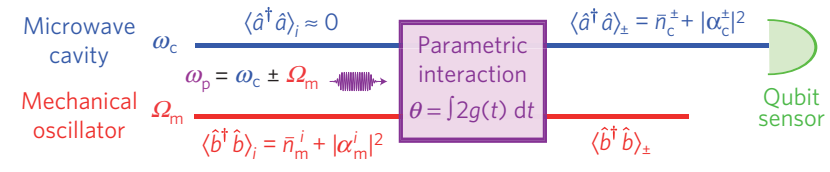

b
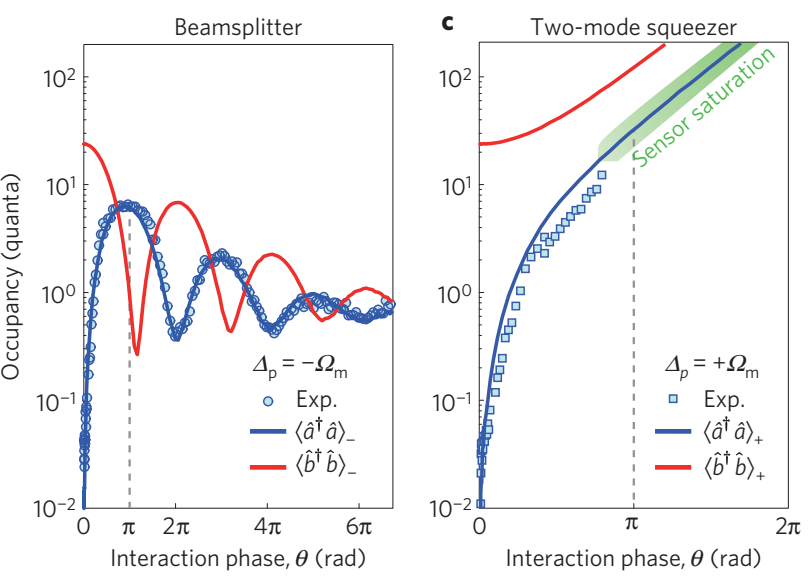

d

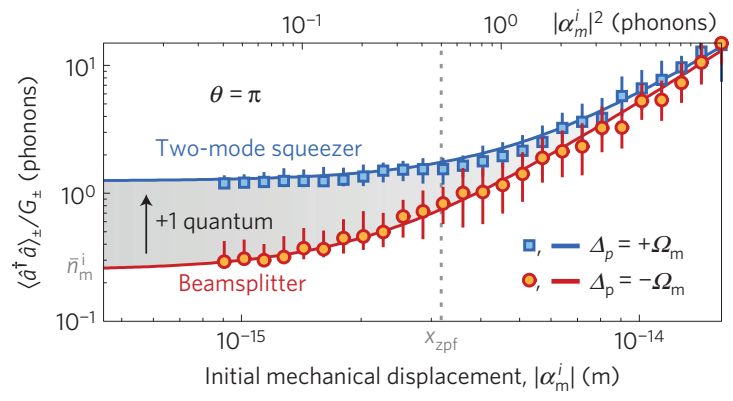

Figure 3 | Optomechanics with a number-resolving detector. a, Sequence diagram. b. Measured cavity occupancy (blue circles) as a function of the interaction duration in reduced unit $\theta$, for $\Delta_{\mathrm{p}}=-\Omega_{\mathrm{m}}\left(n_{\mathrm{p}}=3.8 \times 10^{5}\right.$ and $g=2 \pi \times 198 \mathrm{kHz})$. $\mathbf{c}$, The same as in $\mathbf{b}$ for $\Delta_{\mathrm{p}}=+\Omega_{\mathrm{m}}$. In both $\mathbf{b}$ and $\mathbf{c}$ the predictions of the Heisenberg-Langevin equations of motion are shown in solid blue lines (Supplementary Information). The mechanical occupancy is shown as solid red lines. d, Measurement of the vacuum fluctuations of the mechanical oscillator. The cavity occupancy is measured as a function of the initial mechanical displacement for $\theta=\pi$ and for $\Delta_{\mathrm{p}}= \pm \Omega_{\mathrm{m}}$. The gain at each pump frequency, $G_{ \pm}$, is the ratio of final cavity displacement to initial mechanical displacement $G_{ \pm}=\left|\alpha_{c}^{ \pm}\right|^{2} /\left|\alpha_{m}^{i}\right|^{2}$, which is constant for all $\alpha_{\mathrm{m}}^{i}$. We show $\left\langle\hat{a}^{\dagger} \hat{a}\right\rangle_{-} / G_{-}$in red and $\left\langle\hat{a}^{\dagger} \hat{a}\right\rangle_{+} / G_{+}$in blue. The amplification of the mechanical vacuum fluctuations appears as one additional quantum when $\Delta_{\mathrm{p}}=+\Omega_{\mathrm{m}}$. The error bars are the measurement uncertainty of the photon-number distribution (Supplementary Information).

the thermal, noise-like component of the cavity state from the coherent component.

We will now exploit this measurement technique to explore the out-of-equilibrium optomechanical dynamics (Fig. 3). To acquire some physical intuition one can solve the lossless equations of motion describing the time evolution of the microwave and mechanical field amplitudes (Supplementary Information). The average photon occupancy after a beamsplitter interaction, $\left\langle\hat{a}^{\dagger} \hat{a}\right\rangle_{-}$, or a two-mode squeezer interaction, $\left\langle\hat{a}^{\dagger} \hat{a}\right\rangle_{+}$, follows:

$$
\begin{aligned}
\left\langle\hat{a}^{\dagger} \hat{a}\right\rangle_{-} & =\left\langle\hat{a}^{\dagger} \hat{a}\right\rangle_{i} \cos ^{2}(\theta / 2)+\left\langle\hat{b}^{\dagger} \hat{b}\right\rangle_{i} \sin ^{2}(\theta / 2) \\
\left\langle\hat{a}^{\dagger} \hat{a}\right\rangle_{+} & =\left\langle\hat{a}^{\dagger} \hat{a}\right\rangle_{i} \cosh ^{2}(\theta / 2)+\left\langle\hat{b} \hat{b}^{\dagger}\right\rangle_{i} \sinh ^{2}(\theta / 2)
\end{aligned}
$$

where $\left\langle\hat{a}^{\dagger} \hat{a}\right\rangle_{i}$ and $\left\langle\hat{b}^{\dagger} \hat{b}\right\rangle_{i}$ are respectively the initial cavity and mechanical occupancy, and $\theta=\int 2 g(t) \mathrm{d} t$ is the accumulated 
interaction phase. The periodic functions in equation (1) describe the state exchange induced by the beamsplitter interaction (see Fig. 3b) and the hyperbolic functions in equation (2) describe the amplification induced by the two-mode squeezer interaction (see Fig. 3c). Experimentally, we start by actively preparing the mechanical state in a nearly pure coherent state, $\left\langle\hat{b}^{\dagger} \hat{b}\right\rangle_{i}=\bar{n}_{\mathrm{m}}^{i}+\left|\alpha_{\mathrm{m}}^{i}\right|^{2}$, where $\left|\alpha_{\mathrm{m}}^{i}\right|^{2}=23$ is the coherent component (displacement) and $\bar{n}_{\mathrm{m}}^{i}=0.25$ represents the residual thermal (incoherent) phonon occupancy (Supplementary Information). We then pulse either optomechanical interaction using a microwave pump at $\Delta_{\mathrm{p}}= \pm \Omega_{\mathrm{m}}$, followed by tuning the qubit into resonance with the cavity to measure the subsequent photon-number distribution (as described previously in Fig. 2) for each pump duration. As expected this distribution corresponds to a displaced thermal state, characterized by an incoherent component $\bar{n}_{c}^{ \pm}$and a coherent component $\alpha_{c}^{ \pm}$, for a total average photon occupancy of $\left\langle\hat{a}^{\dagger} \hat{a}\right\rangle_{ \pm}=\bar{n}_{c}^{ \pm}+\left|\alpha_{c}^{ \pm}\right|^{2}$. In Fig. 3b,c, we show $\left\langle\hat{a}^{\dagger} \hat{a}\right\rangle_{ \pm}$as a function of the interaction phase $\theta$. The data in Fig. 3b (Fig. 3c) qualitatively agree with equation (1) (equation (2)), and quantitatively agree with full numerical simulations (solid blue lines) that include the finite linewidth and bath temperature of each mode (Supplementary Information). The expected evolution of $\left\langle\hat{b}^{\dagger} \hat{b}\right\rangle_{ \pm}$follows the solid red line. The only free parameter is the initial mechanical occupancy $\left\langle\hat{b}^{\dagger} \hat{b}\right\rangle_{i}$. We emphasize our ability to resolve, with a sensitivity well below the single-quantum level, the coherent exchange of mechanical phonons and cavity photons or the amplification of the two localized modes, with both processes occurring at a rate faster than decoherence.

A striking signature of the quantum nature of the oscillator's motion resides in the commutation relation $\hat{b} \hat{b}^{\dagger}=\hat{b}^{\dagger} \hat{b}+1$. Together with equation (2), we can see that even with both modes initially in their ground state, $\left\langle\hat{a}^{\dagger} \hat{a}\right\rangle_{i}=\left\langle\hat{b}^{\dagger} \hat{b}\right\rangle_{i}=0$, the zero-point motion of the oscillator alone feeds the parametric amplification process, with a gain $\sinh ^{2}(\theta / 2)$, leading to a finite cavity occupancy $\left\langle\hat{a}^{\dagger} \hat{a}\right\rangle_{+}$. In contrast, from the same initial conditions, equation (1) shows no interesting dynamics for the beamsplitter interaction, with $\left\langle\hat{a}^{\dagger} \hat{a}\right\rangle_{-}=0$. Thus, to quantitatively extract the ' +1 ' contribution during amplification, we compare the two processes, as shown in Fig. 3d. With the cavity initially in its ground state, $\left\langle\hat{a}^{\dagger} \hat{a}\right\rangle_{i}=0$, equation (1) and equation (2) relate the final average cavity occupancy $\left\langle\hat{a}^{\dagger} \hat{a}\right\rangle_{ \pm}$to the initial average mechanical occupancy $\left\langle\hat{b}^{\dagger} \hat{b}\right\rangle_{i}$ through the gain of the parametric interactions, $\sin ^{2}(\theta / 2)$ for the beamsplitter or $\sinh ^{2}(\theta / 2)$ for the two-mode squeezer. First, we set the interaction phase to $\theta=\pi$ and measure the final photon distribution as a function of the initial mechanical displacement $\alpha_{\mathrm{m}}^{i}$, for $\Delta_{\mathrm{p}}=+\Omega_{\mathrm{m}}$ and $\Delta_{\mathrm{p}}=-\Omega_{\mathrm{m}}$. In the presence of loss, both gains are less than their maximum values, $G_{-}<\sin ^{2}(\pi / 2)$ and $G_{+}<\sinh ^{2}(\pi / 2)$. By taking the ratio of final cavity displacement to initial mechanical displacement, $G_{ \pm}=\left|\alpha_{\mathrm{c}}^{ \pm}\right|^{2} /\left|\alpha_{\mathrm{m}}^{i}\right|^{2}$, we measure $G_{-}=0.25$ and $G_{+}=0.88$, at all coherent drive amplitudes (Supplementary Information). We show the results for $\left\langle\hat{a}^{\dagger} \hat{a}\right\rangle_{-} / G_{-}=\left\langle\hat{b}^{\dagger} \hat{b}\right\rangle_{i}$ in red and $\left\langle\hat{a}^{\dagger} \hat{a}\right\rangle_{+} / G_{+}=\left\langle\hat{b}^{\dagger} \hat{b}\right\rangle_{i}+1$ in blue. The difference between these two optomechanical interactions is clear in Fig. $3 \mathrm{~d}$, showing the extra ' +1 ' contribution sourced directly from the commutator between the position and momentum of the macroscopic mechanical oscillator due to the quantum vacuum fluctuations.

This signature has been discussed in terms of asymmetry between the rates of phonon absorption and emission ${ }^{5,7}$, or in terms of added noise in the context of three-wave mixing ${ }^{1,28}$. Our architecture is however uniquely suited to explore quantitatively this phenomenon in optomechanics, because we have the ability to measure directly the mechanically scattered photons, localized in the cavity. By combining the measurement of the intra-cavity photon-number distribution with the optomechanical interactions, we have realized a phonon-number distribution measurement. The nonlinearity of the qubit-cavity interaction allows us to clearly distinguish classical noise from quantum noise, as only classical noise can lead to real cavity quanta that can excite the qubit out of its ground state. In addition, our technique is not sensitive to the correlations between the electromagnetic noise and mechanical noise, which would induce 'squashing' of the output field'.

Looking forward, with more complex protocols, we could: exploit the qubit as a deterministic single-phonon source to generate arbitrary quantum states of motion ${ }^{20}$; perform full-state tomography of the mechanical oscillator ${ }^{29}$. The ability to encode complex quantum states in these long-lived mechanical systems has important implications for quantum information and for the study of the fundamental quantum behaviour of massive objects ${ }^{30}$.

\section{Received 30 January 2015; accepted 11 May 2015;}

published online 15 June 2015

\section{References}

1. Clerk, A. A. et al. Introduction to quantum noise, measurement, and amplification. Rev. Mod. Phys. 82, 1155-1208 (2010).

2. Aspelmeyer, M., Kippenberg, T. \& Marquardt, F. Cavity optomechanics. Rev. Mod. Phys. 86, 1391-1452 (2014).

3. O'Connell, A. D. et al. Quantum ground state and single-phonon control of a mechanical resonator. Nature 464, 697-703 (2010).

4. Jayich, A. M. et al. Cryogenic optomechanics with a $\mathrm{Si}_{3} \mathrm{~N}_{4}$ membrane and classical laser noise. New J. Phys. 14, 115018 (2012).

5. Safavi-Naeini, A. H. et al. Observation of quantum motion of a nanomechanical resonator. Phys. Rev. Lett. 108, 033602 (2012).

6. Safavi-Naeini, A. H. et al. Laser noise in cavity-optomechanical cooling and thermometry. New J. Phys. 15, 035007 (2013).

7. Weinstein, A. J. et al. Observation and interpretation of motional sideband asymmetry in a quantum electromechanical device. Phys. Rev. X 4, 041003 (2014)

8. Teufel, J. D. et al. Sideband cooling of micromechanical motion to the quantum ground state. Nature 475, 359-363 (2011).

9. Palomaki, T. A. et al. Coherent state transfer between itinerant microwave fields and a mechanical oscillator. Nature 495, 210-214 (2013).

10. Palomaki, T. A. et al. Entangling mechanical motion with microwave fields. Science 342, 710-713 (2013).

11. Brune, M. et al. Quantum Rabi oscillation: A direct test of field quantization in a cavity. Phys. Rev. Lett. 76, 1800-1803 (1996).

12. Meekhof, D. M. et al. Generation of nonclassical motional states of a trapped atom. Phys. Rev. Lett. 76, 1796-1799 (1996).

13. Hofheinz, M. et al. Generation of Fock states in a superconducting quantum circuit. Nature 454, 310-314 (2008).

14. Chan, J. et al. Laser cooling of a nanomechanical oscillator into its quantum ground state. Nature 478, 89-92 (2011)

15. Verhagen, E. et al. Quantum-coherent coupling of a mechanical oscillator to an optical cavity mode. Nature 482, 63-67 (2012).

16. Purdy, T. P. et al. Observation of radiation pressure shot noise on a macroscopic object. Science 339, 801-804 (2013).

17. Suh, J. et al. Mechanically detecting and avoiding the quantum fluctuations of a microwave field. Science 344, 1262-1265 (2014).

18. Wallraff, A. et al. Strong coupling of a single photon to a superconducting qubit using circuit quantum electrodynamics. Nature 431, 162-167 (2004).

19. Sillanpaa, M. A. et al. Coherent quantum state storage and transfer between two phase qubits via a resonant cavity. Nature 449, 438-442 (2007).

20. Hofheinz, M. et al. Synthesizing arbitrary quantum states in a superconducting resonator. Nature 459, 546-549 (2009).

21. Pirkkalainen, J. M. et al. Hybrid circuit cavity quantum electrodynamics with a micromechanical resonator. Nature 494, 211-215 (2013).

22. Martinis, J. M. Superconducting phase qubits. Quantum Inf. Process. 8, 81-103 (2009)

23. Teufel, J. D. et al. Circuit cavity electromechanics in the strong-coupling regime. Nature 471, 359-363 (2011)

24. Cicak, K. et al. Low-loss superconducting resonant circuits using vacuum-gap-based microwave components. Appl. Phys. Lett. 96, 093502 (2010)

25. Zakka-Bajjani, E. et al. Quantum superposition of a single microwave photon in two different 'colour' states. Nature Phys. 7, 599 (2011).

26. Hofer, S. G. et al. Quantum entanglement and teleportation in pulsed cavity optomechanics. Phys. Rev. A 84, 052327 (2011). 
27. Law, C. K. \& Eberly, J. H. Arbitrary control of a quantum electromagnetic field. Phys. Rev. Lett. 76, 1055-1058 (1996).

28. Bergeal, N. et al. Analog information processing at the quantum limit with a Josephson ring modulator. Nature Phys. 6, 296-302 (2010).

29. Leibfried, D. et al. Experimental determination of the motional quantum state of a trapped atom. Phys. Rev. Lett. 77, 4281-4285 (1996).

30. Pikovski, I. et al. Probing Planck-scale physics with quantum optics. Nature Phys. 8, 393-397 (2012).

\section{Acknowledgements}

We thank A. W. Sanders for taking the micrographs in Fig. 1b,c. This work was supported by the NIST Quantum Information Program.

\section{Author contributions}

F.L., J.D.T. and R.W.S. conceived and designed the experiment. F.L. fabricated the device and performed the measurement. F.L. and J.D.T. analysed the data. F.L., J.D.T. and R.W.S wrote the manuscript. J.A. contributed to the fabrication process and provided experimental support.

\section{Additional information}

Supplementary information is available in the online version of the paper. Reprints and permissions information is available online at www.nature.com/reprints.

Correspondence and requests for materials should be addressed to F.L.

\section{Competing financial interests}

The authors declare no competing financial interests. 